\title{
Perceptual Limits on the Visual Monitoring Task
}

\author{
Ronald A. Rensink \\ Departments of Psychology and Computer Science, University of British Columbia
}

\begin{abstract}
A brief review is presented of recent work in vision science showing important limits on the ability of observers to carry out various tasks. First, it has been found that only a few moving items can be tracked at any time, even after considerable practice. Second, observers can often miss the appearance of unexpected objects or events, even if these are large. Third, observers can often miss changes that occur in front of them, even when these changes are large, repeated, and expected. Finally, observers simply cannot see two changes at a time, no matter how hard they try. The explanation for these effects is briefly discussed, along with the reasons why these limits are not usually noticed. Some suggestions are also given about what (if anything) can be done to mitigate their effects in regards to the visual monitoring task of the anesthesiologist.
\end{abstract}

\section{INTRODUCTION}

A S observers, we generally have the impression that we can see everything that is happening in front of us. However, recent work in vision science has shown that this impression is incorrect: our ability to accurately perceive our surroundings is far more limited than we generally believe (see e.g., [1]-[4]).

Most of these limits have been found to center around visual attention. In particular, visual attention has been found to be (a) necessary for several aspects of visual perception, and (b) very limited in capacity. Taken together, these findings imply that perceptual limits are likely to be encountered in many (if not most) tasks in everyday life. Owing to the nature of our general environment and the kinds of tasks we perform there, these limits are not usually serious, and our impression of the world can afford to appear accurate and complete. However, these limits are far more serious for tasks that heavily involve visual attention, and that are carried out in specialized environments that are not all that forgiving. Such limits are believed to be the cause of accidents in specialized activities such as driving [5]. It is argued here that these limits are also potentially relevant for the visual monitoring carried out in anesthesiology.

This paper will focus on those limits most relevant to the visual monitoring task. Since these limits are usually either not perceived or else greatly underestimated [6], one goal of this paper is to increase general awareness of their existence. The other goal is to begin discussion of the countermeasures that can be taken to reduce their incidence and their effects.

Manuscript received April 21, 2008. This work was supported in part by the Natural Sciences and Engineering Research Council of Canada.

R. A. Rensink is with the departments of Computer Science and Psychology, University of British Columbia, Vancouver, BC V6T 1 Z4 Canada. (phone: 604-822-2579; fax: 604-822-6923; e-mail: rensink@ cs.ubc.ca or rensink@psych.ubc.ca).

\section{TYPES OF PERCEPTUAL LIMITS}

\section{A. Tracking Limits}

An important issue in vision science is the extent to which an observer can track moving items in space. The displays used for these tests usually consist of about a dozen items moving at constant speed on a blank background; the observer is asked to monitor a selected subset [7].

Results indicate that only 3-4 items can be successfully tracked, even after extensive practice. This has been taken to indicate that (a) attention is necessary to track an item, and (b) attentional capacity is limited to 3-4 items (an estimate consistent with values obtained using other techniques).

Further experiments have shown that this limit is reduced even further as the speed of the items is increased [8]. In addition, other kinds of experiment have shown that attentional capacity decreases with age [9], suggesting that older observers will have greater difficulty in tracking more than a few items at a time.

Finally, the graphical representations used in the display can also potentially lower the effectiveness of tracking. For example, it has been found that if pairs of disks are connected with lines, the observer will treat each connected pair as a basic item, tracking only the centers of these pairs, and not the individual disks [10].

\section{B. Inattentional Blindness}

Recent work in vision science has shown that when an observer attends to a given task, they will often miss the appearance of unexpected objects and events, a phenomenon known as inattentional blindness [4]. This suggests that visual attention is needed for perception; without it, an observer can be blind to objects and events, even when these are large and potentially relevant. For example, over half of observers missed an unexpected appearance of a person in a gorilla suit, who walked across the entire screen [11].

Inattentional blindness is found in real-life situations; among other things, it is believed to be a leading cause of automobile accidents, especially when attention is distracted by things such as cell-phone use [5]. It is also found in head-up displays, where two images are superimposed; if an event occurs in the unattended channel, it will often be missed, even when the observer is looking right at it [12].

It is also important to note that inattentional blindness may still occur, even if the object or event is expected. The critical factor is not expectation, but attention: if the attention of an observer is strongly enough engaged on a task, other items can effectively become unseeable, even when the observer is looking right at them [13]. 


\section{Change Blindness}

Another important phenomenon is the failure to notice changes in a display, even when the objects themselves are seen, and the changes are large and expected. This is known as change blindness; it is believed to indicate that visual attention is necessary to see change [1]-[3].

Results indicate that only 3-4 items can be seen to change at any time; this limit appears to remain even after extensive practice. This likely reflects an attentional capacity similar to that found in tracking (and in other attentional tests).

Change blindness occurs whenever the observer fails to attend the changing item at the moment of the change. To prevent it, therefore, the observer must therefore continually attend the relevant item. This is difficult in practice: change blindness can potentially occur if the change is made at the moment an observer moves their eyes, or even blinks [2].

\section{Change Simultanagnosia}

An important concern in regards to visual monitoring is change simultanagnosia, the inability of an observer to see more than one change occur at a time, even if attending to the relevant items [14].

This phenomenon appears to be due to the pooling of the information that is attended. If several changes occur at the same, their contents are therefore pooled together, and the individual components cannot be separated out. Only when changes occur sequentially can the components be perceived as such. Consequently, when several changes occur simultaneously (within a fraction of a second), the first one may be picked up. But if the others are similar, they may be washed out by the idempotent nature of the change detection operation, and effectively lost.

\section{BLINDNESS TO THESE LIMITS}

\section{A. Virtual Representation}

Although perceptual limits of the kind described here can be severe, failures of visual perception are not generally noticed. For example, most people are subject to change blindness blindness, i.e., the belief that they are far better at detecting changes than they actually are [6].

One possible explanation for this is that our perceptual systems usually work quite well in spite of these limits. It has been proposed that our perception of our surroundings is supported by a virtual representation, in which attention is given to the right object at the right time [2], [15], [16]. The management of attention required is based on several assumptions about the world, such as the relatively scarcity of change [15], [16]. But while appropriate for our ancestral environment, these assumptions may not be true of more specialized environments in modern life.

\section{B. Confabulation}

Another possible reason for the general lack of awareness of these perceptual limits is the nature of the conscious mind itself. More precisely, the visual picture experienced by an observer is the result of a great deal of processing, and tends to be edited to be consistent with existing belief structures (see, e.g., [17]). In other words, observers often don $t$ see something until they believe it. If they don $t$ believe that they have limits on their perceptual abilities, they simply will not experience them.

\section{COUNTERMEASURES}

Although our perceptual abilities may suffer from serious limits, we are not obliged to simply accept this situation. To begin with, even the simple awareness of these limits would likely motivate observers to be more careful. Although perhaps not a feasible strategy in general, it could certainly be applied on occasion in specialized tasks, such as the visual monitoring done by anesthesiologists.

Second, visual displays might be designed to take these limits into account, and mitigate their worst effects. Indeed, if designed to work with the mechanisms used by the visual system, such displays may one day even enable visual performance beyond that found in everyday life [16], [18].

\section{REFERENCES}

[1] R. A. Rensink, J. K. O’Regan, and J. J. Clark. To see or not to see: The need for attention to perceive changes in scenes, Psychol. Science, vol. 8, pp. 368-373, 1997.

[2] R. A. Rensink, Change Detection, Annual Review of Psychology, vol. 53, pp. 245-277, 2002.

[3] D. J. Simons, Change Blindness and Visual Memory, East Sussex, UK: Taylor \& Francis, 2000.

[4] A. Mack, and I. Rock, Inattentional Blindness, Cambridge MA: MIT Press, 1998.

[5] D. L. Strayer, F. A. Drews, and W. A. Johnston, Cell phone-induced failures of visual attention during simulated driving, Journal of Experimental Psychology: Applied, vol. 9, pp. 23-32, 2003.

[6] D. T. Levin, N. Momen, S. E. Drivdahl, and D. J. Simons. Change blindness blindness: The metacognitive error of overestimating change-detection ability, Vis. Cognition, vol. 7, pp. 397-412, 2000.

[7] Z. W. Pylyshyn, R. W. Storm, Tracking multiple independent targets: Evidence for a parallel tracking mechanism, Spatial Vision, vol. 3, pp. 179-197, 1988.

[8] G. Liu, et al., Multiple-Object Tracking Is Based on Scene, Not Retinal, Coordinates, Journal of Experimental Psychology: Human Perception and Performance, vol. 31, pp. 235-247, 2005.

[9] C. J. Harp, and R. A. Rensink, A comparison of attentional processing in younger and older observers, Invest. Ophthalmol \& Visual Science, vol 40, p. 50, 1999.

[10] B. J. Scholl, Z. W. Pylyshyn, and J. Feldman, What is a visual object? Evidence from target merging in multiple object tracking, Cognition, vol. 80, pp. 159-177, 2001.

[11] D. J. Simons, and C. F. Chabris, Gorillas in our midst: Sustained inattentional blindness for dynamic events, .Perception, vol 28, pp. 1059-1074, 1999.

[12] U. Neisser, U., and R. Becklen, Selective looking: Attending to visually significant events, Cog. Psychology, vol 7, 480-494, 1975.

[13] R. A. Rensink. (2005). Robust Inattentional Blindness, J. Vision [Online] vol 5, p. 790a. Available: http://journalofvision.org/5/8/790.

[14] R. A. Rensink. (2002). Failure to see more than 1 change at a time J. Vision [Online], vol 2, p.245a, http://journalofvision.org/2/7/245.

[15] R. A. Rensink, The dynamic representation of scenes, Vis. Cognition, vol. 7, pp. 17-42, 2000.

[16] R. A. Rensink, The Modeling and Control of Visual Perception, in Integrated Models of Cognitive Systems, W. Gray Ed. New York: Oxford University Press, 2007, pp. 132-148.

[17] P. Johansson, L. Hall, S. Sikstr m, and A. Olsson, Failure to Detect Mismatches Between Intention and Outcome in a Simple Decision Task, Science, vol. 310, pp. 116-119, 2005.

[18] R. A. Rensink, Internal vs. external information in visual perception, in Proc. 2nd Intl. Symposium on Smart Graphics, Hawthorne, NY, 2002, pp. 63-70. 